\title{
Adaptation of Full-Reference Image Quality Assessment Methods for Automatic Visual Evaluation of the Surface Quality of 3D Prints
}

\author{
Krzysztof Okarma*, Jaroslaw Fastowicz \\ Department of Signal Processing and Multimedia Engineering, Faculty of Electrical Engineering, \\ West Pomeranian University of Technology in Szczecin, \\ Sikorskiego 37, 70-313 Szczecin, Poland \\ okarma@zut.edu.pl
}

\begin{abstract}
Automatic visual quality assessment of the 3D printed surfaces is currently one of the most demanding challenges in additive manufacturing. Regardless of the applications of the computer vision for the 3D printing process monitoring purposes, a reliable surface quality evaluation during manufacturing may introduce brand new possibilities. The detection of some distortions and their automatic evaluation can be helpful when deciding to stop the process to save time, energy, and filament. In some cases, some further corrections can also be made for relatively small distortions. Since many general-purpose image quality assessment methods have been proposed in recent years, their applications for the quality evaluation in the additive manufacturing are investigated. As most of the metrics are full-reference and require the availability of the original perfect quality image, their direct application is not possible. Therefore, their adaptation is described in the paper together with experimental verification of classification results obtained using various metrics.
\end{abstract}

Index Terms-Additive manufacturing; 3D prints; Surface quality assessment; Image analysis.

\section{INTRODUCTION}

The additive manufacturing technology, also referred as $3 \mathrm{D}$ printing, becomes one of the most revolutionary technology in modern industry, being one of the fundaments of the development of the Industry 4.0 solutions [1]. Such fourth industrial revolution brings together interdisciplinary areas of science and technology related to electronics, automation, robotics, mechatronics, IT, telecommunication, as well as electrical engineering and transport. Regardless of the idea of smart factories, a relevant type of non-massive production supplementing the market needs and necessities, may be the use of 3D printing technology, which becomes more and more popular. The growing availability of $3 \mathrm{D}$ printers makes possible to design and produce many individual objects, e.g., mechanical parts, as well as rapidly build prototypes or even some unique tools [2].

The application areas of the additive manufacturing are still growing, including biomedical engineering, preserving the cultural heritage and art, visualization of various $3 \mathrm{D}$

Manuscript received 6 March, 2019; accepted 3 July, 2019. objects or model making. One of the most typical applications might be the 3D scanning of some old used elements and then "copying" them using additive manufacturing technology for the replacement purposes [3].

Since 3D printing is a considerably slow process, there is often an obvious necessity of the continuous monitoring of the manufacturing progress, as well as the quality monitoring of the produced objects [4]. In case of failure detection, the printing process should be stopped. However, when the lower surface quality is found, an appropriate action might be aborting the whole process to save filament, energy, and time or - for smaller distortions - trying to correct the surface in the last step of manufacturing. It is worth noting that surface quality assessment may also be related with the mechanical properties of the manufactured element although, in most cases, the main reason of such an evaluation is aesthetic.

In this paper, some opportunities of using image analysis for the automatic visual surface quality estimation of $3 \mathrm{D}$ prints are analyzed, particularly in a view of possible application of the general-purpose full-reference (FR) image quality metrics proposed during recent several years. Although their direct application is impossible because of the lack of the reference images, they can be efficiently used for the comparison of image fragments. Since the high values of image quality assessment (IQA) measures usually indicate the high surface quality, their decrease is typically caused by the existence of some structural deformations locally visible in one of the compared image fragments.

\section{MACHINE ViSION IN ADDITIVE MANUfACTURING}

Most of the approaches proposed by various researchers utilizing machine vision in $3 \mathrm{D}$ printing focus on the process monitoring. One of the first of such solutions was proposed by Fang et al. [5], [6], where so called process signatures were used to detect failures in ceramics printing. Another idea of the inspection of top surface and boundaries of layers during printing was presented later by Cheng and Jafari [7]. Comparing the consecutive layers before the next one is deposited, 2D profiles from the representative signatures were obtained useful for detection of the volume voids. The 
main purpose of the method developed by Szkilnyk et al. [8] was the detection of failures in automated assembly machines using the machine vision approach based on processing od images captured by webcams in the LabVIEW environment. Further extensions of this idea were discussed by Chauhan and Surgenor [9], [10].

Recently, an interesting inspection system for 3D printers was proposed in the reference [11] that allows detection of collisions with the use of machine learning and tracking based on singular points. Scime and Beuth [12] used unsupervised machine learning method with the use of image patches for an automatic detection and classification of anomalies occuring during the spreading of the powder in the laser powder bed fusion process. On the other hand, Delli and Chang [13] recently used a supervised machine learning approach for process monitoring. Tourloukis et al. [14] used neural networks to analyze the quality of electronic products obtained using the 3D inkjet printing technology. Holzmond and $\mathrm{Li}$ [15] compared the geometry of the 3D printed object using the 3D digital image correlation with the computer model to detect print defects.

The application of image analysis of the images captured by cameras in the system build from five Raspberry Pi units for in-situ detection of defects was proposed by Straub [4]. Although the system was able to detect the "dry printing" and premature job termination, its main disadvantages were high sensitivity to camera movements and illumination changes. Nevertheless, some possibilities of using the machine vision algorithms even for limited computational power systems were demonstrated. Another successful attempt to the application of machine vision in 3D printing was Multifab project [16] initially presented at SIGGRAPH 2015 conference. In this multi-material 3D printing solution, captured images were used for self-calibration of the printheads and 3D scanning, as well as close-feedback loop useful for corrections of prints. As stated by the Authors, the overall price of the system should not exceed $\$ 7,000$.

Another recent solution [17] is related with the detection of stripes caused by motions of printheads without printing with leaking drops of filament. The Authors proposed the use of the ant colony optimization to choose the tool path speeding-up the manufacturing process and avoiding some of the visual artifacts.

Nevertheless, none of the discussed solutions utilized the image quality assessment approach to describe numerically the overall quality of the printed surfaces to make a decision about continuing or interrupting the manufacturing process.

\section{SELECTED FULL-REFERENCE IMAGE QUALITY ASSESSMENT METHODS}

During recent several years, a significant progress in objective image quality assessment can be observed. It changed from the pixel based comparison based on Mean Squared Error (MSE) and Peak Signal to Noise Ratio (PSNR) or similar formulas into much more sophisticated comparisons of image fragments and/or features. The first metric changing the approach to the objective FR IQA methods was the Universal Image Quality Index (UIQI) proposed by an active group of researchers under the leadership of Alan Conrad Bovik from the Laboratory for Image \& Video Engineering (LIVE) located within the University of Texas at Austin in 2002 [18]. It became a basic point for the popular Structural Similarity (SSIM) metric [19] and its multi-scale version (MS-SSIM) proposed by the same group several months later [20]. The formula of the local SSIM for the $11 \times 11$ pixels fragments of images $x$ and $y$ based on average values, variances and covariance was defined as follows

$$
\operatorname{SSIM}(x, y)=\frac{\left(2 \overline{x y}+C_{1}\right)\left(2 \sigma_{x y}+C_{2}\right)}{\left(\bar{x}^{2}+\bar{y}^{2}+C_{1}\right)\left(\sigma_{x}^{2}+\sigma_{y}^{2}+C_{2}\right)},
$$

where $C_{1}=(0.01 \times L)^{2}$ and $C_{2}=(0.03 \times L)^{2}$ are small constants used to avoid possible divisions by zero for the darkest and/or flat areas of images and $L=256$ is the number of permitted levels in grayscale images. The final quality index is then obtained as the average for all positions of the sliding window and the local similarity values form the quality map. It is also worth noting that most of IQA metrics were defined only for the single channel images and quality assessment of the color images is still a challenge.

During the next years, several modifications of these methods were proposed by various researchers, including the Gradient SSIM [21], Quality Index based on Local Variance (QILV) [22], Riesz based Feature Similarity (RFSIM) [23] or Feature Similarity (FSIM) [24]. Some other proposed metrics utilized the Singular Value Decomposition, eg., RSVD [25] or the information theory, eg., Information Fidelity Criterion (IFC), as well as the Visual Information Fidelity (VIF) [26], [27]. The source codes of MATLAB implementations of many metrics can be found on the webpages of their Authors and some of them were collected in the MeTriX_MuX package [28].

To increase the correlation of objective assessment results with subjective evaluations, some hybrid/combined metrics utilizing multi-metric fusion approach were proposed, including the first such nonlinear fusion of MS-SSIM, VIF, and R-SVD [29], as well as CISI metric [30] being the weighted product of MS-SSIM, VIF, and FSIMc. Next combination with RFSIM and improved FSIMc metric led to the Extended Hybrid Image Similarity (EHIS) metric [31], further increasing the linear correlation coefficient with subjective scores available in IQA databases.

Unfortunately, all these metrics, as well as many other approaches to general-purpose IQA utilizing the comparisons of the reference "pristine" images and their distorted versions (full-reference methods), cannot be directly applied for the assessment of 3D printed surfaces, especially during the printing process, due to the lack of reference image.

Nevertheless, some preliminary attempts to the off-line application of selected metrics assuming the division of 3D printed surface images into smaller fragments were presented in earlier papers [32], [33]. Obtained results were promising. However, the verification of results was made for a limited number of images obtained by a 2D flatbed scanner in controlled lighting conditions and the application of these methods for a larger number of photos did not lead to 
satisfactory results. Therefore, further improvement of this approach towards its potential in-situ application in 3D printing devices, including the verification of the usefulness of some other IQA metrics, is the main motivation of the research presented in this paper.

\section{PROPOSED APPROACH AND EXPERIMENTAL RESUlTS}

The quality assessment of 3D printed surfaces, particularly conducted during the manufacturing process, can be efficiently made assuming the cameras observing the side of the manufactured object. Considering the principle of operations of the most popular Fused Deposition Modelling (FDM) based devices, the filament is deposited layer by layer from the printing head and, therefore, the consecutive layers should be well visible for a side view. Usually, two types of thermoplastic filaments are used in such a low cost 3D printers - Polyactic Acid (PLA) and Acrylonitrile Butadiene Styrene (ABS) with slightly different physical properties, e.g., melting temperature.

Due to the expected regularity of the visible layers for high quality prints, high image similarity values should be obtained when individual images representing fragments of the same surface are compared. As a result of some local distortions, the similarity factors should decrease since, in practical applications, these contaminations will occur in different locations in each fragment of the compared images. They can be related with "dry printing" (lack of the filament or under-filling), presence of cracks (especially for ABS filament), as well as over-filling. Such distortions may be caused by improper melting temperature, inappropriate speed of the filament's delivery, problems with stepper motors, etc.). To verify the suitability of the proposed approach, several planar samples were manufactured employing three available devices (Prusa i3, RepRap Pro Ormerod 2, and da Vinci 1.0 Pro 3-in-1) that use various colors of PLA and ABS filaments. The exemplary obtained samples are shown in Fig. 1.

All samples captured by cameras were subjectively assessed by the colleagues from our department and classified as high, moderately high, moderately low, and low quality. The main goal of the considered method is the classification of the samples in accordance with subjective evaluation.
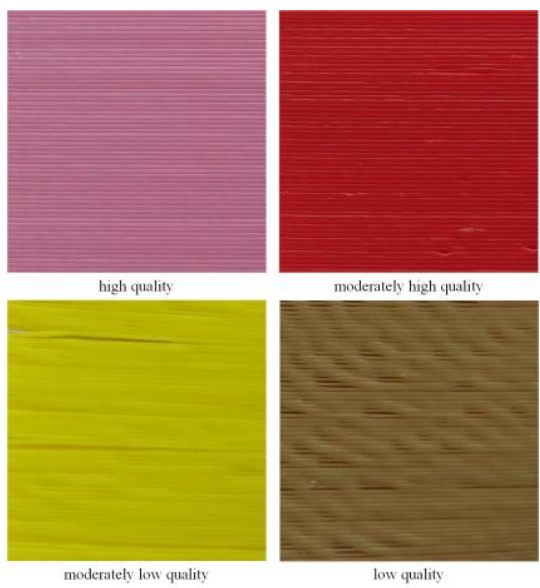

Fig. 1. Examples of high, moderately high, moderately low, and low quality $3 \mathrm{D}$ printed samples.

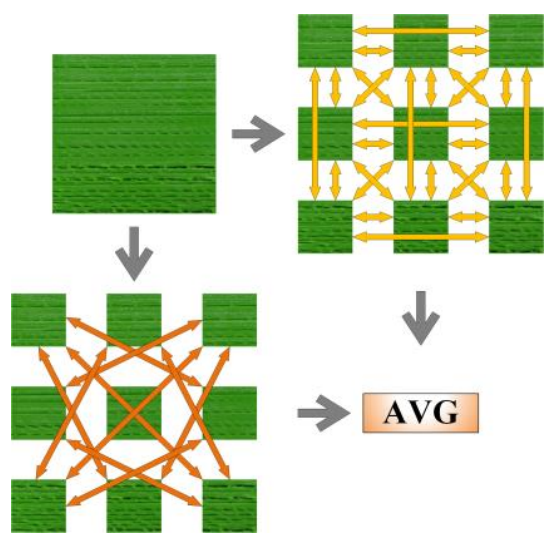

Fig. 2. Illustration of the idea of the mutual similarity calculation between 9 image fragments.

Since all considered FR IQA metrics were developed for a single channel images, the obtained photos were converted to grayscale using the ITU Recommendation BT.601-7 implemented in MATLAB rgb2gray function. Additionally, the contrast adjustment operation was conducted to suppress the impact of image brightness on the obtained results.

The next operation influencing significantly both the results and the further computation time, is the division of the image into fragments. To limit the number of comparisons, the division into 4, 9, and 16 fragments were used. The idea of the division into 9 fragments obtained as the pattern of $3 \times 3$ blocks is presented in Fig. 2 .

For the division into 4 fragments $(2 \times 2$ blocks pattern), only 6 mutual similarities have to be calculated, whereas using 9 fragments requires the calculation of 36 similarities as shown in Fig. 2. Using the division into 16 fragments denotes 120 such calculations and, therefore, in such case, the computational complexity of the used metric may play a significant role. So, in practical on-line applications, the use of some metrics, which require more computations, particularly combined ones, should be restricted to lesser number of blocks. However, in some cases, depending on hardware possibilities, the local similarity calculations might be partially done in parallel.

Nevertheless, since the division of an image into smaller fragments may cause the phase shift between the compared fragments, the additional phase adjustment is proposed based on the calculation of the 1D correlation between both compared fragments in vertical direction (after averaging of all pixels' values in each row). The adjustment of the phase shift to maximize the calculated correlation with cropping both images ensures that the top and bottom rows of compared fragments represent the same phase of the different layers (e.g., the darkest lines of layers). Since the resolution of the captured images is $1600 \times 1600$ pixels, the height of each layer of the filament is several pixels (depending on the nozzle size used during the manufacturing).

The experimental verification of usefulness of the proposed approach was done using several FR IQA metrics with the division into 4,9 , and 16 fragments with the phase adjustments as described above. Considering the relatively long computation time of some of the metrics (e.g., VIF, RSVD or combined metrics), we focused on similarity metrics with typical processing time less than one second for the 
proposed algorithm using the MATLAB environment installed on a PC with $17-7700 \mathrm{HQ}(2.8 \mathrm{GHz})$ processor and 16 GB RAM.

As can be seen analyzing Fig. 3-Fig. 6 illustrating the experimental results obtained using some of the investigated metrics, the application of most of them (e.g., PSNR and SSIM) even for 16 blocks does not lead to appropriate classification. All the colors in the plots represent the colors of individual samples and the symbols representing the quality are the same as shown in the legend of Fig. 3.

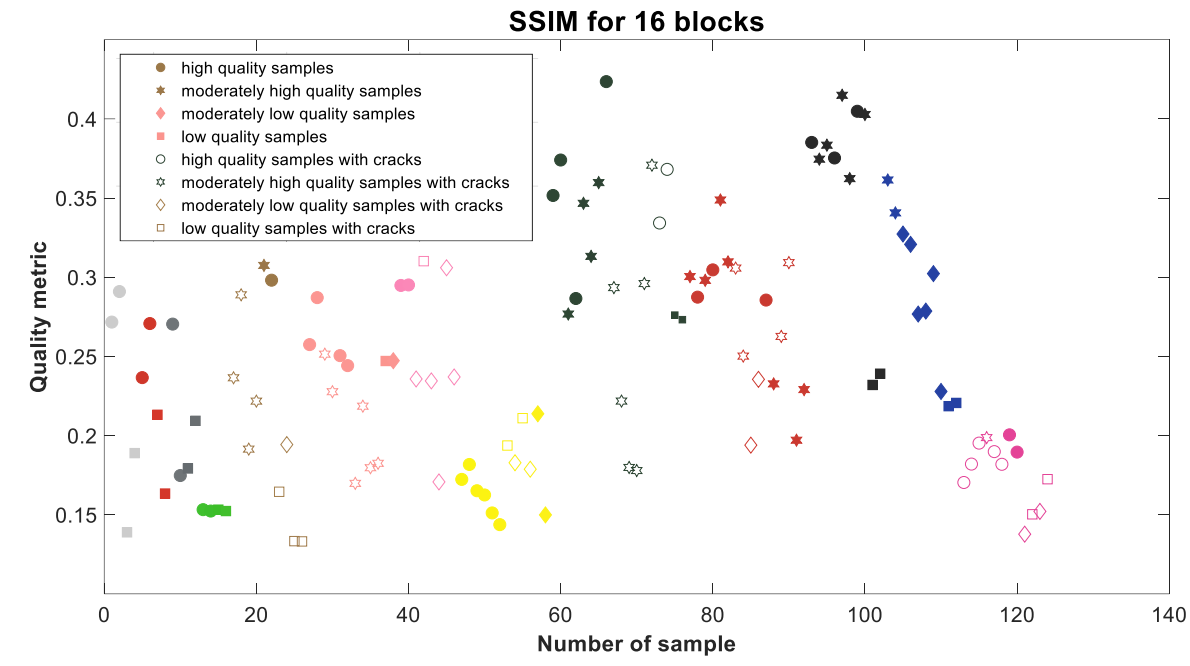

Fig. 3. Obtained results for the application of SSIM metric using 16 blocks.

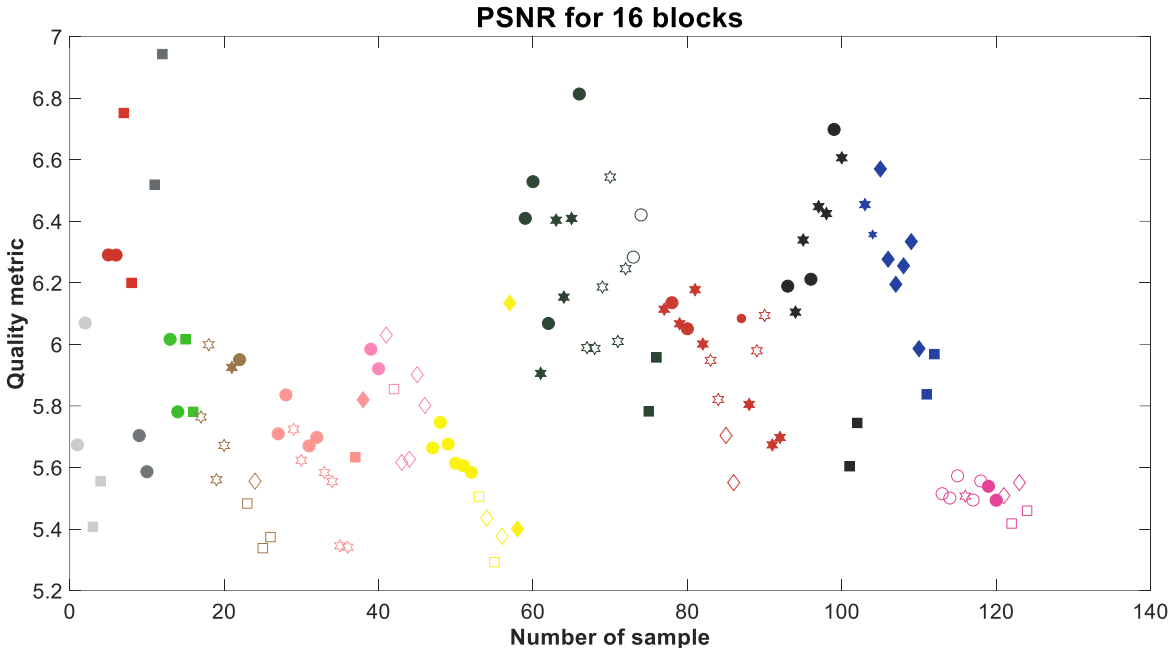

Fig. 4. Obtained results for the application of PSNR metric using 16 blocks.

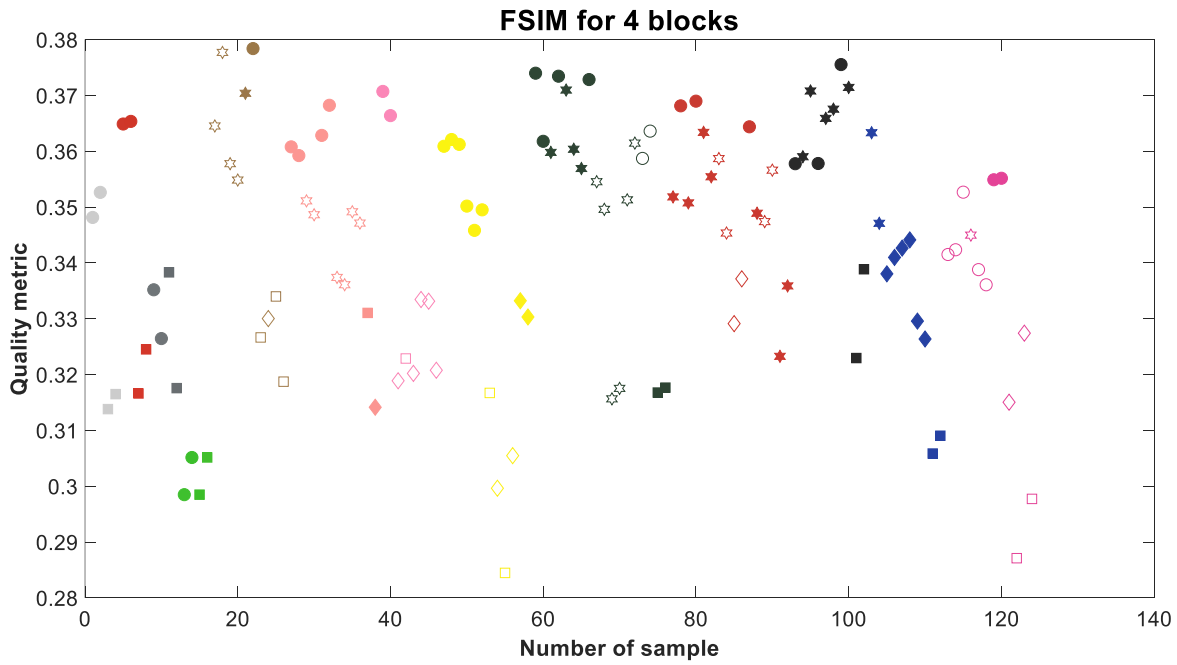

Fig. 5. Obtained results for the application of FSIM metric using 4 blocks. 


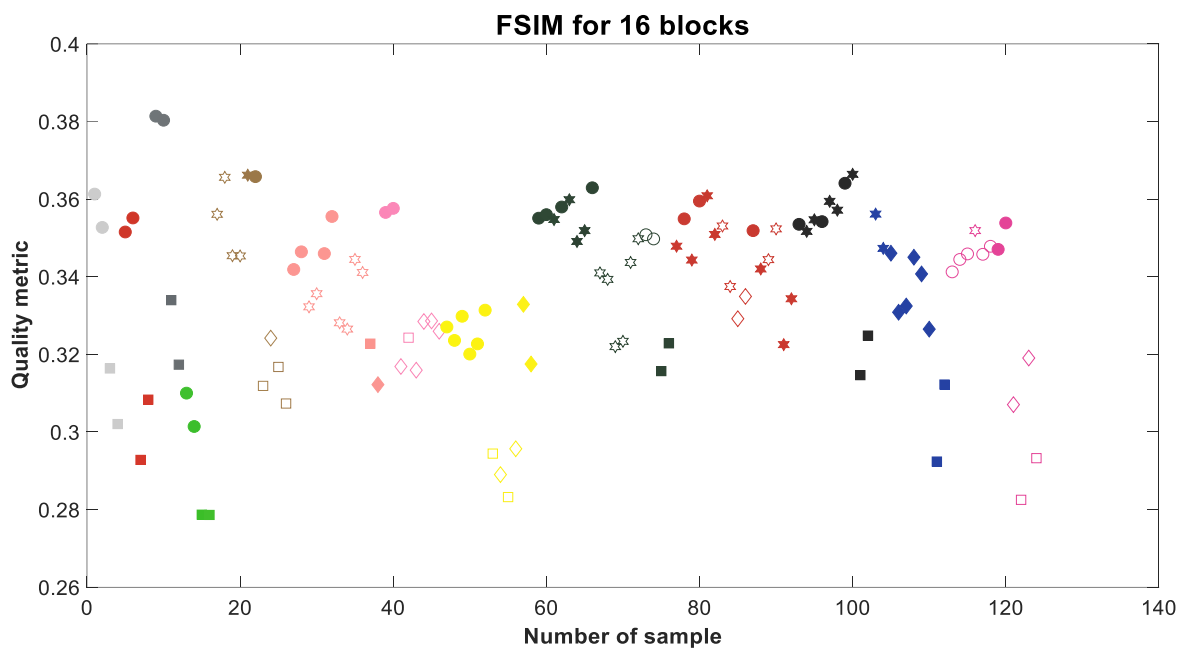

Fig. 6. Obtained results for the application of FSIM metric using 16 blocks.

The best results were achieved for the use of the Feature Similarity with the division of the images into 16 blocks. As illustrated in Fig. 6, for each color of the filament analyzed separately, some improper classifications (assuming the division into two general sets - one for high and moderately high quality samples and the second for low and moderately low ones) took place only for some of the ABS filaments: one yellow sample (No. 57), one dark green sample (No. 76), and two red samples (No. 85 and No. 86 or No. 91 and No. 92 depending on the assumed threshold).

Therefore, for improperly classified 4 samples out of 124 , the overall accuracy reached $96,8 \%$. Considering the high quality samples as "true" and low quality as "false", depending on the chosen threshold for the red samples, we obtained 2 false positives (FP) and 2 false negatives (FN) or only $4 \mathrm{FP}$ samples. For that reason, the obtained sensitivity is 0.975 , whereas the specificity is equal to 0.954 (or 0.913 with the sensitivity equal to 1 considering all the improperly classified samples as false positives). Regardless of the choice of the threshold for red samples, the obtained FMeasure, typically used for evaluation of the classification, is 0.975 outperforming all of the other considered image quality metrics. For example, with the use of SSIM (for results shown in Fig. 3), 18 improperly classified samples were obtained lowering the accuracy to 0.855 and the FMeasure to 0.896 .

It is worth noting that all the experiments for images were done captured by camera and, therefore, they cannot be directly compared with the previous results obtained for partially the same samples scanned using the 2D flatbed scanner. Considering the controlled lighting conditions and uniform lighting of the sample surfaces during the 2D scanning process, the values of the metrics were more independent on the illumination changes between the fragments of the images and, therefore, the categorization into high and low quality samples was easier.

\section{CONCLUSIONS}

The application of general-purpose full reference image quality metrics for the objective assessment of 3D printed surfaces is a challenging task. Due to relatively long processing time, some of the IQA methods calculated utilizing the proposed approach based on mutual similarity of image fragments, cannot be used efficiently in on-line

\section{applications.}

Some of the analyzed metrics, e.g., PSNR or RFSIM, lead to improper classification and, therefore, they cannot be used for the objective evaluation of 3D printed surfaces.

Nevertheless, the application of the FSIM metric for the proposed approach makes it possible to properly classify most of the tested samples. The obtained results are comparable to previously obtained results for a smaller testing dataset, which turned out to be less appropriate for some colors of the filaments (particularly ABS). The achieved accuracy is promising and confirms the usefulness of the FR IQA metrics for the automatic quality estimation of $3 \mathrm{D}$ printed surfaces assuming the prior knowledge of the filament's color, which can be gathered during the precalibration process.

Future development of the proposed approach will be related to the combination with some other investigated methods utilizing image entropy and line detection using Hough transform of HOG features. Nevertheless, extensive experiments would be necessary to decrease the necessary computational efforts and provide the metric correlated with subjective evaluation. This task will require gathering the subjective quality scores from numerous observers, similarly as in the general-purpose IQA databases.

\section{REFERENCES}

[1] S. Chong, G.-T. Pan, J. Chin, P. L. Show, T. C. K. Yang, and C.-M Huang, "Integration of 3D printing and Industry 4.0 into engineering teaching", Sustainability, vol. 10, no. 11, p. 3960, 2018. DOI: $10.3390 /$ su 10113960 .

[2] A. Gebhardt, J. Kessler, and L. Thurn. 3D Printing. understanding additive manufacturing, $2^{\text {nd }}$ Edition, Carl Hanser Verlag $\mathrm{GmbH} \&$ Co. KG, Munich, Germany, 2018. DOI: 10.3139/9781569907030.

[3] R. Jiang, R. Kleer, and F. T. Piller, "Predicting the future of additive manufacturing: A Delphi study on economic and societal implications of 3D printing for 2030", Technological Forecasting and Social Change, vol. 117, pp. 84-97, 2017. DOI: 10.1016/j.techfore.2017.01.006.

[4] J. Straub, "Initial work on the characterization of additive manufacturing (3D printing) using software image analysis", Machines, vol. 3, no. 2, pp. 55-71, 2015. DOI 10.3390/machines3020055.

[5] T. Fang, M. A. Jafari, I. Bakhadyrov, A. Safari, S. Danforth, N Langrana, "Online defect detection in layered manufacturing using process signature", in Proc. IEEE Int. Conf. Systems, Man and Cybernetics, San Diego, CA, USA, 1998, vol. 5, pp. 4373-4378. DOI: 10.1109/ICSMC.1998.727536.

[6] T. Fang, M. A. Jafari, S. C. Danforth, and A. Safari, "Signature 
analysis and defect detection in layered manufacturing of ceramic sensors and actuators", Machine Vision and Applications, vol. 15, no. 2, pp. 63-75, 2003. DOI: 10.1007/s00138-002-0074-1.

[7] Y. Cheng and M. A. Jafari, "Vision-based online process control in manufacturing applications", IEEE Trans. Automation Science and Engineering, vol. 5, no. 1, pp. 140-153, 2008. DOI 10.1109/TASE.2007.912058.

[8] G. Szkilnyk, K. Hughes, and B. Surgenor, "Vision based fault detection of automated assembly equipment", in Proc. ASME/IEEE Int. Conf. Mechatronic and Embedded Systems and Applications, Washington, DC, USA, 2011, Parts A and B, vol. 3 pp. 691-697. DOI: 10.1115/DETC2011-48493.

[9] V. Chauhan and B. Surgenor, "A comparative study of machine vision based methods for fault detection in an automated assembly machine", Procedia Manufacturing, vol. 1, pp. 416-428, 2015. DOI: 10.1016/j.promfg.2015.09.051.

[10] V. Chauhan and B. Surgenor, "Fault detection and classification in automated assembly machines using machine vision", The Int. J. Advanced Manufacturing Technology, vol. 90, no. 9, pp. 2491-2512, 2017. DOI: 10.1007/s00170-016-9581-5.

[11] N. G. Makagonov, E. M. Blinova, and I. I. Bezukladnikov, "Development of visual inspection systems for 3D printing", in Proc. of 2017 IEEE Conference of Russian Young Researchers in Electrical and Electronic Engineering (EIConRus), 2017, pp. 14631465. DOI: 10.1109/EIConRus.2017.7910849.

[12] L. Scime and J. Beuth, "Anomaly detection and classification in laser powder bed additive manufacturing process using a trained computer vision algorithm", Additive Manufacturing, vol. 19, pp. 114-126, 2018. DOI: 10.1016/j.addma.2017.11.009.

[13] U. Delli and Sh. Chang, "Automated process monitoring in 3D printing using supervised machine learning", Procedia Manufacturing, vol. 26, pp. 865-870, 2018. DOI: 10.1016/j.promfg.2018.07.111.

[14] G. Tourloukis, S. Stoyanov, T. Tilford, and C. Bailey, "Data driven approach to quality assessment of 3D printed electronic products", in Proc. of 38th Int. Spring Seminar on Electronics Technology (ISSE), 2015, pp. 300-305. DOI: 10.1109/ISSE.2015.7248010.

[15] O. Holzmond, X. Li, "In situ real time defect detection of 3D printed parts", Additive Manufacturing, vol. 17, pp. 135-142, 2017. DOI: 10.1016/j.addma.2017.08.003.

[16] P. Sitthi-Amorn, J. E. Ramos, Y. Wang, J. Kwan, J. Lan, W. Wang, and W. Matusik, "Multifab: A machine vision assisted platform for multi-material 3D printing", ACM Trans. Graph., vol pp. 34, no. 4, 129:1-129:11, 2015. DOI: 10.1145/2766962.

[17] K. Y. Fok, Ch.-Ts. Cheng, N. Ganganath, H. H.-Ch. Iu, and Ch. K. Tse, "An ACO-based tool-path optimizer for 3D printing applications", IEEE Trans. Industrial Informatics, 2018. DOI: 10.1109/TII.2018.2889740.

[18] Z. Wang and A. Bovik, "A universal image quality index", IEEE Signal Processing Letters, vol. 9, no. 3, pp. 81-84, 2002. DOI: 10.1109/97.995823.

[19] Z. Wang, A. Bovik, H. Sheikh, and E. Simoncelli, "Image quality assessment: From error measurement to structural similarity", IEEE Trans. Image Processing, vol. 13, no. 4, pp. 600-612, 2004. DOI: 10.1109/TIP.2003.819861

[20] Z. Wang, E. Simoncelli, and A. Bovik, "Multi-scale structural similarity for image quality assessment", in Proc. of 37th IEEE Asilomar Conf. Signals, Systems and Computers, Pacific Grove, California, 2003, vol. 2, pp. 1398-1402. DOI: 10.1109/ ACSSC.2003.1292216.

[21] G.-H. Chen, Ch.-L. Yang, Sh.-L. Xie, "Gradient-based Structural Similarity for image quality assessment", in Proc. of IEEE Int. Conf. on Image Processing ICIP, Atlanta, Georgia, 2006, pp. 2929-2932. DOI: 10.1109/ICIP.2006.313132.

[22] S. Aja-Fernández, R. San-José-Estépar, C. Alberola-López, and C. Westin, "Image quality assesment based on local variance", in Proc. of 28th Annu. IEEE Int. Conf. Engineering in Medicine and Biology Society EMBS, New York, 2006, pp. 4815-4818. DOI: 10.1109/IEMBS.2006.259516.

[23] L. Zhang, L. Zhang, and X. Mou, "RFSIM: A feature based image quality assessment metric using Riesz transforms", in Proc. of 17th IEEE Int. Conf. on Image Processing ICIP, Hong Kong, China, 2010, pp. 321-324. DOI: 10.1109/ICIP.2010. 5649275

[24] L. Zhang, L. Zhang, X. Mou, and D. Zhang, "FSIM: A feature similarity index for image quality assessment", IEEE Trans. Image Processing, vol. 20, no. 8, pp. 2378-2386, 2011. DOI: 10.1109/TIP.2011.2109730.

[25] A. Mansouri, A. Mahmoudi-Aznaveh, F. Torkamani-Azar, and J. A. Jahanshahi, "Image quality assessment using the singular value decomposition theorem", Optical Review, vol. 16, no. 2, pp. 49-53, 2009. DOI: 10.1007/s10043-009-0010-y.

[26] H. Sheikh, A. Bovik, and G. de Veciana, "An information fidelity criterion for image quality assessment using natural scene statistics", IEEE Trans. on Image Processing, vol. 14, no. 12, pp. 2117-2128, 2005. DOI: 10.1109/TIP.2005. 859389.

[27] H. Sheikh, A. Bovik, "Image information and visual quality", IEEE Trans. on Image Processing, vol. 15, no. 2, pp. 430-444, 2006. DOI: 10.1109/TIP.2005.859378

[28] M. Gaubatz, MeTriX MuX visual quality assessment package [Online]. Available: http://foulard.ece.cornell.edu/gaubatz/metrix_mux/

[29] K. Okarma, "Combined full-reference image quality metric linearly correlated with subjective assessment", in ICAISC (1), ser. Lecture Notes in Computer Science, L. Rutkowski, R. Scherer, R Tadeusiewicz, L. A. Zadeh, and J. M. Zurada, Eds., Springer, 2010, vol. 6113, pp. 539-546. DOI: 10.1007/978-3-642-13208-7_67.

[30] K. Okarma, "Combined image similarity index", Optical Review, vol. 19, no. 5, pp. 249-254, 2012. DOI: 10.1007/s10043-012-0055-1.

[31] K. Okarma, "Extended hybrid image similarity - combined fullreference image quality metric linearly correlated with subjective scores", Elektronika ir Elektrotechnika, vol. 19, no. 10, pp. 129-132, 2013. DOI: 10.5755/j01.eee.19.10.5908.

[32] K. Okarma, J. Fastowicz, and M. Tecław, "Application of structura similarity based metrics for quality assessment of 3D prints", in Proc. of Computer Vision and Graphics: International Conference, ICCVG 2016, ser. Lecture Notes in Computer Science, L. J. Chmielewski, A. Datta, R. Kozera, K. Wojciechowski, Eds., Springer International Publishing, 2016, vol. 9972, pp. 244-252. DOI: 10.1007/978-3-31946418-3_22.

[33] J. Fastowicz, K. Okarma, "Fast quality assessment of 3D printed surfaces based on structural similarity of image regions", in Proc. 2018 Int. Interdisciplinary PhD Workshop, Świnoujście, Poland, 2018, pp. 401-406. DOI: 10.1109/IIPHDW.2018.8388399. 